\title{
An Energy Efficient Routing Protocol based on Periodic Route Discovery for Mobile Ad Hoc Networks
}

\author{
Ch. Balaswamy ${ }^{1}$ K. Naga Prakash ${ }^{2}$ and Ch. Hima Bindu ${ }^{3}$ \\ ${ }^{1}$ Department of Electronics and Communication Engineering, QIS College of Engg \& \\ Technology, Ongole, India \\ ch.balaswamy 7 @gmail.com \\ ${ }^{2}$ Department of Electronics and Comupters Engineering, \\ K L University, Vijayawada, India \\ karatapuprakash. @yahoo.com \\ karatapuprakash@yahoo.com \\ ${ }^{3}$ Department of Electronics and Communication Engineering, QIS College of Engg \& \\ Technology, Ongole, India \\ hb. muvvala@gmail.com
}

\begin{abstract}
In present energy efficient protocols for mobile ad hoc networks, once the route is selected in the route discovery phase, the selected route is used until all the data packets are sent out or until the selected route fails due to the exhaustion of node battery energy. Due to the continuous flow of the packets through a selected route, and if a node in that route having less battery energy, then that node will certainly die out causing route failure and this may also cause failure of other routes which are connected to it. The problem being the energy level at this node is consumed and hence may lead to failure. To reduce this problem, a mechanism of periodic route discovery is introduced. In this mechanism, the route discovery process is reinitialized periodically to increase the network lifetime. It is observed from our simulation results that the proposed mechanism named "PRD based - MMBCR" performs better than the existing energy efficient protocols.
\end{abstract}

\section{KEYWORDS}

Ad hoc network, Routing Protocol, Energy saving, Route discovery and Energy Efficient

\section{INTRODUCTION}

Issues related to saving energy can be found on each protocol layer in ad hoc networks. At the data link layer power can be saved by reducing control messages, which are used to assert neighbour relationships and synchronization purposes. By reducing the amount of control messages sent in MAC protocols, power will be saved, but at the expense of increased delays. When control messages are sent frequently the topology will be also less known, requiring this to be discovered first, incurring longer delays. The control messages can also be used to power of the transmitter at suitable points in time. Unsurprisingly, designing good protocols with few packet collisions will reduce power consumption since retransmission of packets requires energy. At the network layer, the routing protocols can be designed such that there is an increase in the network lifetime by attempting to distribute the forwarding load over multiple different paths. The main objective of this paper is to investigate the performance of the energy efficient routing protocols for mobile ad hoc networks. In this paper, the problem of routing in a wireless ad-hoc network from the viewpoint of energy efficiency is considered. Each node in a wireless ad-hoc network runs on a local energy source which has a limited energy life span. Thus, energy conservation is a critical issue in such networks. To maximize the lifetime of nodes in a 
network, the energy consumption rate of each node must be evenly distributed. Section 2 analyses the existing energy efficient routing protocols. Section 3 presents the proposed mechanisms to increase the network lifetime. Section 4 describes about the experimental results and lastly, section 5 gives the conclusion.

\section{Theoritical Analysis of Energy Efficient Routing Protocols}

Analysis of the energy efficient routing protocols is carried out by using an example network as shown in fig. 1. Energy Efficient protocols consider the remaining energies of the nodes while selecting a route to the destination. In Minimum Battery Cost Routing (MBCR) protocol, since the route cost function is sum of the individual cost functions of the nodes, a route with less energized node may be selected which is a demerit. Unlike MBCR, the Min-Max Battery Cost Routing (MMBCR) first finds the node having minimum battery capacity in each of the possible routes and selects the route having the maximum value among the selected routes. That means the route having maximum lifetime is selected. But the main demerit of MMBCR is that it doesn't consider the transmission powers of the nodes. In MMBCR, the updated information is not considered for route selection. So, two mechanisms are proposed to overcome this disadvantage. The first is MMBCR-route reply, where the cost functions are calculated in route reply phase instead of in route request phase for selecting the route. The other is MMBCR with periodic route discovery to get more updated information about the routes. In this method, periodically the route discovery process is done. If there are any changes in the route, the route information is updated. Because of this method, different routes are used for the transmission of data packets and periodic shifting between the routes which avoids the over usage of nodes and node exhaustion leading to the increase of the lifetime of the network.

\section{Proposed Routing Mechanisms}

At first, the algorithms are developed to implement the energy efficient protocols and proposed mechanisms using NS-2 Network Simulator [6] in the environment of Red Hat Linux-9. The information about NS-2 is given in the website referenced in [7].

\subsection{MMBCR-Route Reply}

In existing MMBCR, the cost functions are calculated and stored in the route request packet header while the route request packets are going from source to destination. To get more updated information of the cost functions, it is better if the cost functions are calculated and stored in route reply packet header while coming back from the destination to source. This technique is proposed in MMBCR-route reply.

In MMBCR, the decision of the route selection is made by the destination. Here, the destination waits for some time to collect all the route requests (RREQs). Also after making the decision, it takes some time for the route reply (RREP) to reach the source. In between changes may occur in the energies of the nodes in the network. Hence the updated information is not considered in MMBCR for route selection. This can be overcome by calculating the cost functions in route reply phase instead of in route request phase for the selection of the route.

In MMBCR-Route Reply, the decision is made by the source node. The destination node simply replies to all the RREQs that reach it. Let the source node initialized the route request. The intermediate nodes simply forward the RREQ packet. The destination node replies immediately after it receives the RREQ packet to the corresponding route. In the route reply phase, the 
intermediate nodes calculate their cost functions, record the value in the RREP packet and follow the same method as in MMBCR route request phase. The source node waits for some time and receives all the RREP packets and selects the route with maximum lifetime and sends the data packets through the route. Because of this method, the updated information about the nodes is known and the best route is selected. One more advantage of this mechanism is that since the source node receives all possible routes from the destination, it stores these routes in the cache for further use which is not in the case of MMBCR where the source receives only one route from the destination.

\subsection{PRD based - MMBCR}

The other problem in the existing MMBCR protocol is that once the route is selected in the route discovery phase, the selected route is used until all the data packets are sent out or until the selected route fails due to node's battery exhaust. If any node in the selected route with less energy, that node will certainly die out causing route failure and hence the total network lifetime. Here, nodes in the selected route suffer lot continuously due to the packets coming from other nodes. To reduce this problem, a mechanism of periodic route discovery is introduced in which the route discovery process is initialized periodically to increase the network lifetime.

\section{EXPERIMENTAL RESULTS}

\subsection{Route failure time of MBCR}

Consider the network as shown in Fig. 1, according to the trace file generated in the NS2 simulator, the energy levels of all the nodes are Node $0-1.301571$, Node $1-1.016685$, Node 2 - 1.238781, Node $3-1.073581$, Node $4-0.620541$, Node $5-1.218352$ and Node $6-$ 1.301747. The corresponding cost functions are Node $0-0.768620$, Node $1-0.983654$, Node 4 -1.611672 , Node $5-0.820880$, Node $2-0.807393$, Node $3-0.931826$. The total cost function along the route $0-4-5$ is 3.201172 and the total cost function along the route $0-1-2-3-6$ is 3.491493. Here, the route 0-4-5 has minimum total cost in the sense it has maximum battery capacity compared to the route 0-1-2-3-6. Since MBCR selects the route with minimum total cost (with maximum battery capacity), the route 0-4-5 is selected as shown in Fig. 1. But, the disadvantage of the MBCR is that even though there is minimum battery cost in the route i.e., 0 4-5-6 is used, since this route has a node 4 which has maximum battery cost, this route will die out early as shown in the Fig. 2Author names are to be written in $13 \mathrm{pt}$.

\subsection{Route failure time of MMBCR}

Consider the same network as shown in Fig. 2, according to the trace file generated in the NS2 simulator, the energy levels of all the nodes are Node $0-1.301571$, Node $1-1.016685$, Node 2 - 1.238781, Node $3-1.073581$, Node $4-0.620541$, Node $5-1.218352$, and Node $6-$ 1.301747. The corresponding cost functions are Node $0-0.768620$, Node $1-0.983654$, Node 4 -1.611672 , Node $5-0.820880$, Node $2-0.807393$, Node $3-0.931826$. The maximum cost function along the route $0-4-5-6$ is 1.611672 and the total cost function along the route $0-1-2-3-$ 6 is 0.983654 . The route $0-4-5-6$ is avoided since it consists of a node with minimum battery capacity compared to the route 0-1-2-3-6. Hence, MMBCR selects the route 0-1-2-3-6 which has higher battery capacity node as shown in Fig. 2. Since the MMBCR protocol avoids a node with the maximum battery cost, the route 0-4-5-6 is avoided and the route 0-1-2-3-6 is selected. This selected route will live longer in comparison with the route failure time in MBCR. 


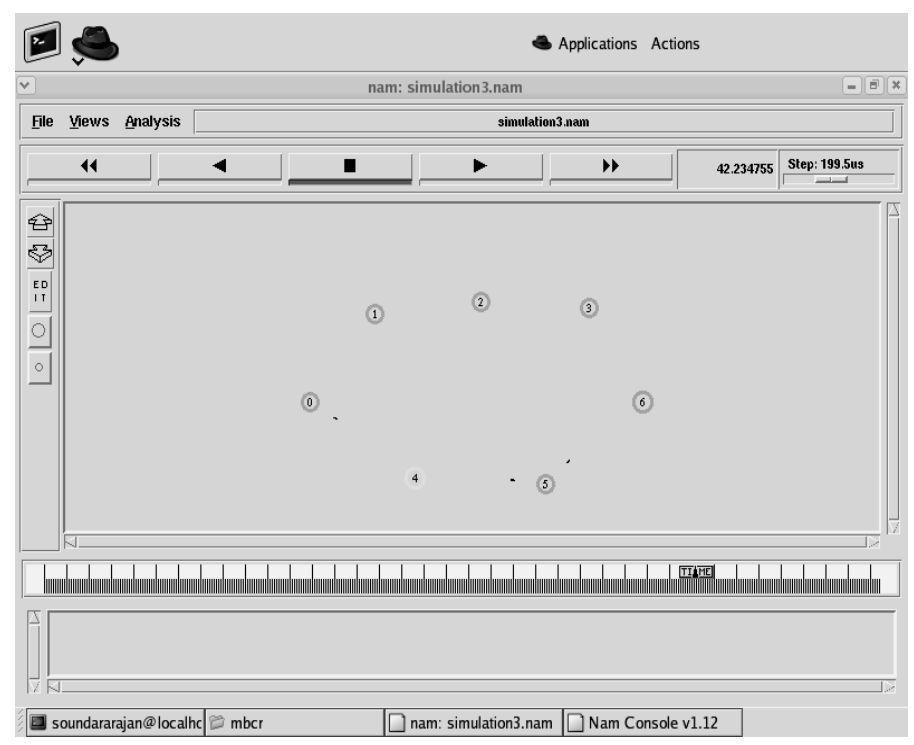

Fig. 1 A snapshot showing the route 0-4-5-6 is selected by MBCR

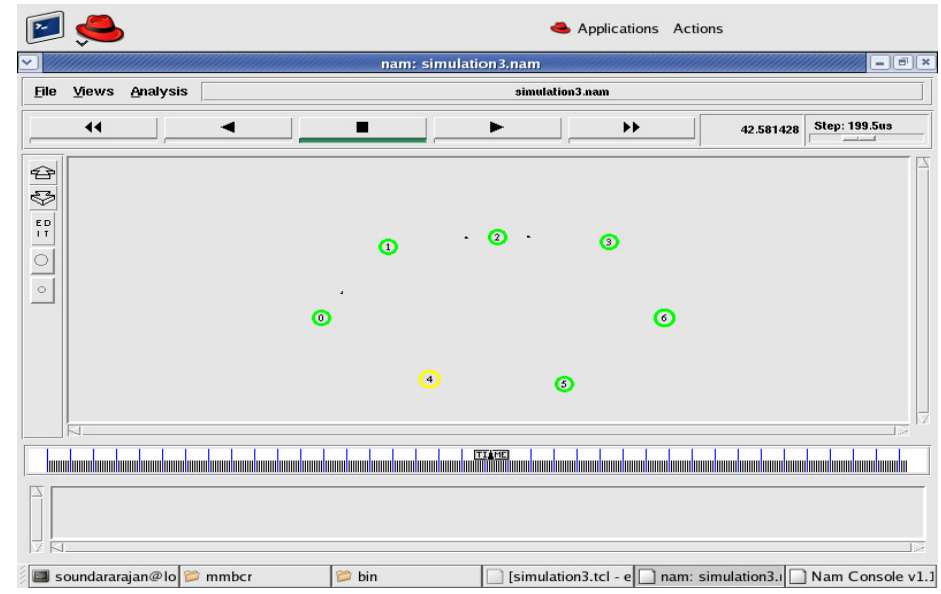

Fig. 2 A snapshot showing the route 0-1-2-3-6 is selected by MMBCR

\subsection{Route failure time of PRD based - MMBCR}

The proposed routing protocol PRD based-MMBCR will activate the route discovery process at periodic times, the burden on a single route is avoided, and many packets will be sent by the source node and the route failure time extends compared to both the MBCR and MMBCR. The results of the route failure times of MBCR, MMBCR and PRD based -MMBCR using NS2 simulator are shown in the Fig. 3.

\subsection{Optimum value for Route discovery period in PRD based - MMBCR}

The simulations are carried out for the network shown in Fig. 1 to investigate the optimum value. In the proposed 'PRD based - MMBCR' protocol, the route discovery period ' $\lambda$ ' is 
defined as the number of packets sent before the route discovery is reinitialized to find new route in which the battery energy is more in comparison with other possible routes.

The simulations are carried out to investigate the optimum value of $\lambda$. The Fig. 4 shows the graph between $\lambda$ and node failure time, and the Fig. 5 shows the graph between $\lambda$ and routing overhead. By observing the results, it is noticed for the value of $\lambda$ equal to 10 where the route discovery is reinitialized for every 10 packets, the node failure time is less due to more routing overhead. As the $\lambda$ increases to 50, the node failure time also increases as shown in figure 4 due to decrease in the routing overhead as shown in Fig. 5. It is observed that for large values of $\lambda$, even though the routing overhead decreases the node failure time decreases. This is because of the overuse of a single route to forward more number of packets which in turn decreases the battery energy of each node in that route and a node which is having less energy dies quickly. From the above observations, at $\lambda$ equal to 50 , the node failure time is high for reasonable routing overhead which is approximately equal for high values of $\lambda$ and hence the value of $\lambda$ equal to 50 is observed as an optimum value.

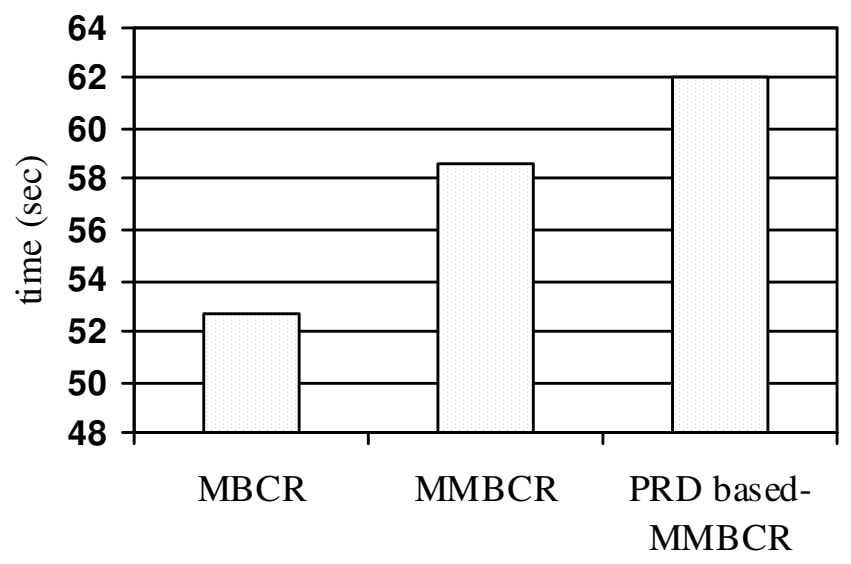

Fig. 3 Comparison of Route Failure times of MBCR, MMBCR and PRD based-MMBCR 


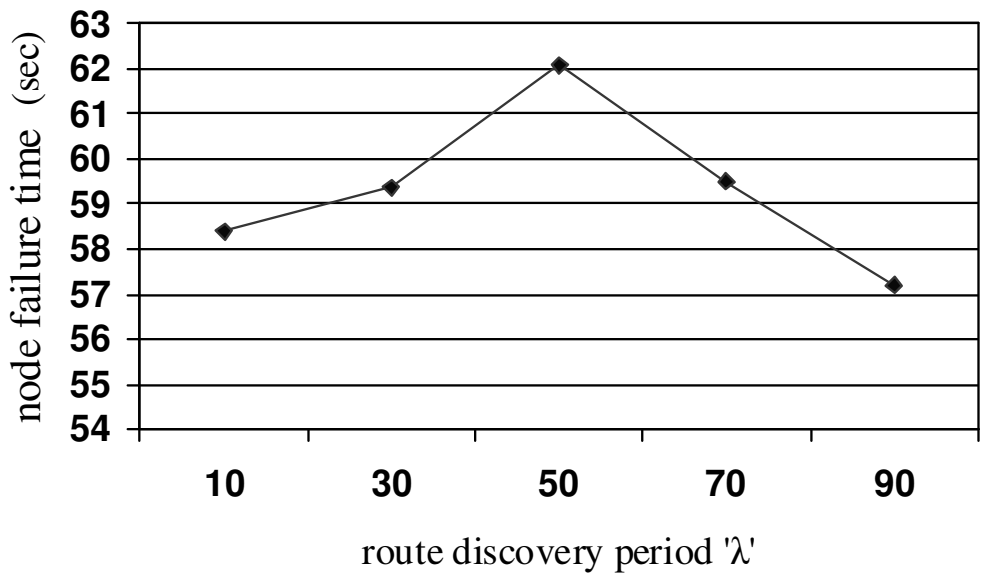

Fig. 4 Node failure times for periodic route discovery in PRD based-MMBCR

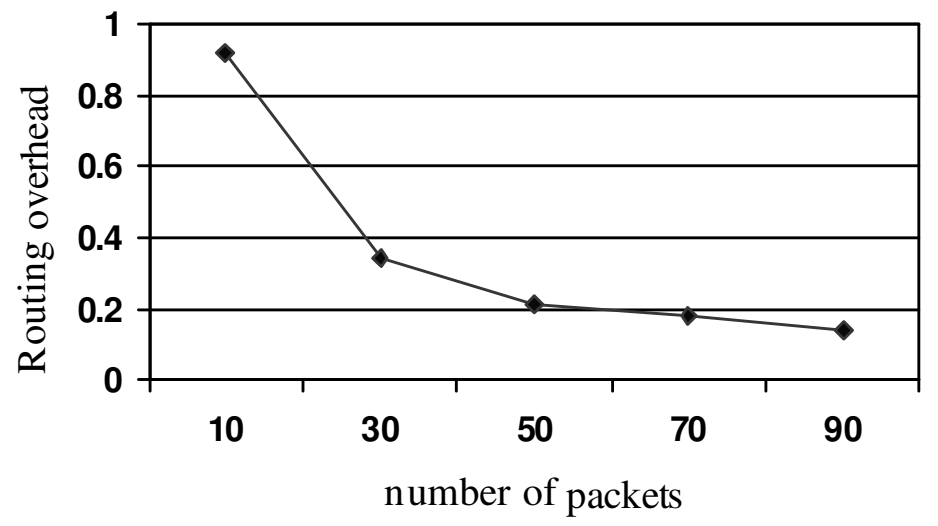

Fig. 5 Routing overhead for periodic route discovery in PRD based-MMBCR

\section{CONCLUSIONS}

In this paper, two mechanisms are proposed to increase the network lifetime. The first is MMBCR-route reply, where the cost functions are calculated in route reply phase instead of in route request phase for the selecting the route. The other is MMBCR with periodic route discovery to get more updated information about the routes. In this method, periodically the route discovery process is done. If there are any changes in the route, the route information is updated. Because of this method, different routes are used for the transmission of data packets and periodic shifting between the routes which avoids the over usage of nodes and node exhaustion leading to the increase of the lifetime of the network. The algorithms for the existing energy efficient protocols and the proposed mechanisms are developed and implemented in NS2 environment. The simulation results show that the proposed mechanism PRD based MMBCR performs better than present MBCR and MMBCR in case of first node failure time. 
For a reasonable routing overhead, an optimum period is investigated for PRD based - MMBCR to get higher node failure time.

\section{REFERENCES}

[1] Mohammed Tarique, Kemal E. Tepe, and Mohammad Naserian, "Energy Saving Dynamic Source Routing for Ad Hoc Wireless Networks", Int. Proc. Of WIOPT, 2005.

[2] S. Singh and C.S. Raghavendra, "PAMAS-Power Aware Multi-Access Protocol with signalling for Ad Hoc Networks", ACM Commn. Rev., July 1998.

[3] S. Singh, M. Woo, and C.S. Raghavendra, " Power Aware routing in Mobile Ad Hoc Networks, “Proc. Mobicom' 98, Dallas, TX, Oct 1998.

[4] C.K. Toh, "Maximum Battery Life Routing to support ubiquitous Mobile computing in Wireless Ad Hoc Networks", IEEE Communications Magazine, June 2001.

[5] W. Cho and S.L. Kim, "A fully distributed routing algorithm for maximizing lifetime of a wireless ad hoc network, " $4^{\text {th }}$ Int. Workshop on Mobile and Wireless Communications Network, 2002, Sep 2002, pp. 670-674

[6] The Network Simulator NS-2, http://www.isi.edu/nsnam/ns/

[7] http://www.isi.edu/nsnam/ns/tutorial

\section{Authors}

Ch. Balaswamy received the B.E degree in Electronics and Communication Engineering from S.R.K.R. Engineering College, Bhimavaram in 1998. He received the M.Tech degree in Electronics and Communication Engineering from Malnad College of Engineering, Hassan, India in 2001. He worked as assistant professor in various engineering colleges in Andhra Pradesh, India. He worked as associate professor in Lakireddy Balireddy College of Engineering, Krishna district, India from August 2005 to October 2006. He received his Ph.D from JNTU, Anantapur in 2010, Now, he is with QIS College of Engineering and Technology, Ongole as Professor and Head of the Electronics and Communication Department. His area of interest is mobile Ad hoc networks and Embedded systems.

K. Naga Prakash received B.E degree in Electronics and Communication Engineering from Nagarjuna University. He received the M.Tech degree in Computer Applications from VT University, India in 2001. He worked as assistant professor in Visvodaya Institute of Technology and Science, Kavali, for 3 years and in LBR College of Engineering for 4 years. He worked as Associate Professor in HITECH College of Engineering, Hyderabad for 3 years and in KL University since Dec 2009. Presently he is pursuing Ph. D under JNT University in the area of Signal Processing.

Ch. Hima bindu is currently working as Associate Professor in ECE Department, QIS College of Engineering \& Technology, ONGOLE, India. She is working towards her Ph.D. at JNTUK, Kakinada, India. She received her M.Tech. from the same institute. She has eight years experience of teaching undergraduate students and post graduate students. She has published two research papers in International journal and more than two research papers in National \& International Conferences. Her research interests are in the areas of image Segmentation, image Feature Extraction and Signal Processing.
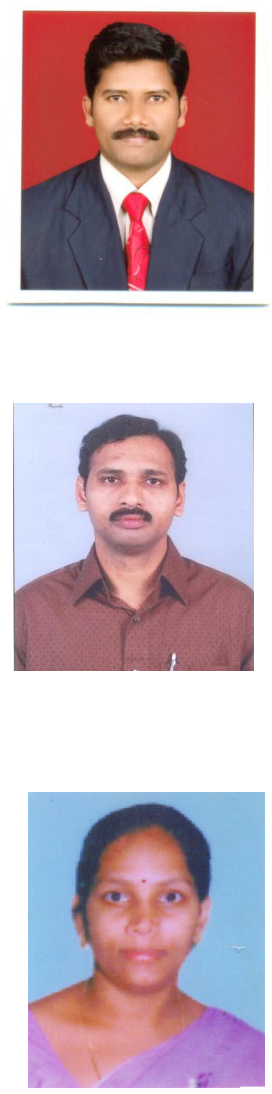